\title{
Comparing transcriptome profiles of human embryo cultured in closed and standard incubators
}

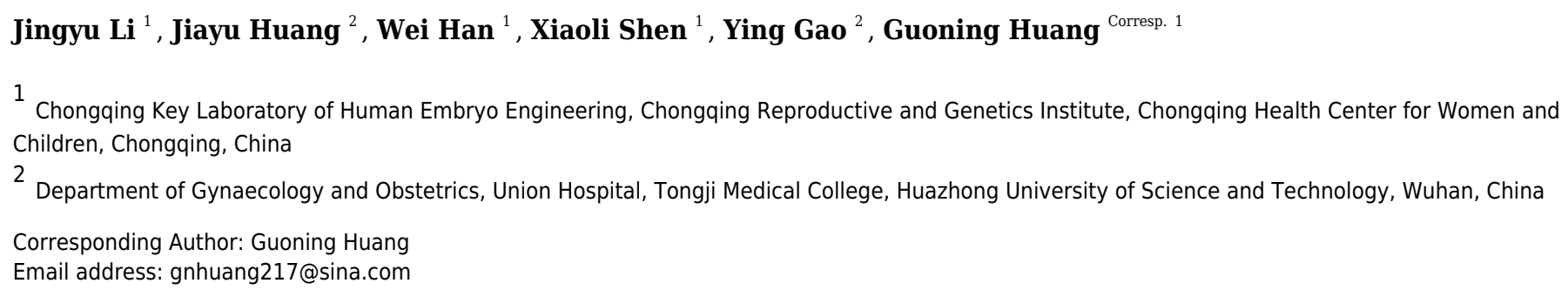

It is necessary to compare the transcriptomic profiles of human embryos cultured in timelapse imaging incubators (TLI) and standard incubators (SI) in order to determine whether a closed culture system has a positive impact on embryos. In this study, we used RNAsequencing (RNA-Seq) to characterize and compare the gene expression profiles of eightcell embryos of the same quality grade cultured in TLI and SI. We sequenced a total of $580,952,620$ reads for zygotes, TLI-cultured, and SI-cultured eight-cell embryos. The global transcriptomic profiles of the TLI embryos were similar to those of the SI embryos and were highly distinct from the zygotes. We also detected 539 genes showing differential expression between the TLI and SI groups with a false discovery rate (FDR) $<0.05$. Using gene ontology (GO) enrichment analysis, we found that the highly expressed SI genes tended to execute functions such as transcription, RNA splicing, and DNA repair, and that the highly expressed TLI genes were enriched in the cell differentiation and methyltransferase activity pathways. This study, the first to use transcriptome analysis to compare SI and TLI, will serve as a basis for assessing the safety of TLI application in assisted reproductive technology (ART). 
1 Comparing transcriptome profiles of human embryo cultured in closed and standard

2

3

4

5 Jingyu Li ${ }^{1}$, Jiayu Huang ${ }^{2}$, Wei Han ${ }^{1}$, Xiaoli Shen ${ }^{1}$, Ying Gao ${ }^{2}$, Guoning Huang ${ }^{1 *}$

6

$7{ }^{1}$ Chongqing Key Laboratory of Human Embryo Engineering, Chongqing Reproductive and

8 Genetics Institute, Chongqing Health Center for Women and Children, Chongqing, China

$9 \quad{ }^{2}$ Department of Gynecology and Obstetrics, Union Hospital, Tongji Medical College, Huazhong

University of Science and Technology, Wuhan, China

11

12

13

14

15

16

17

\section{incubators}

Running title: Comparing closed and standard incubators 


\section{Abstract}

It is necessary to compare the transcriptomic profiles of human embryos cultured in time-lapse imaging incubators (TLI) and standard incubators (SI) in order to determine whether a closed culture system has a positive impact on embryos. In this study, we used RNA-sequencing (RNASeq) to characterize and compare the gene expression profiles of eight-cell embryos of the same quality grade cultured in TLI and SI. We sequenced a total of 580,952,620 reads for zygotes, TLIcultured, and SI-cultured eight-cell embryos. The global transcriptomic profiles of the TLI embryos were similar to those of the SI embryos and were highly distinct from the zygotes. We also detected 539 genes showing differential expression between the TLI and SI groups with a false discovery rate $(\mathrm{FDR})<0.05$. Using gene ontology $(\mathrm{GO})$ enrichment analysis, we found that the highly expressed SI genes tended to execute functions such as transcription, RNA splicing, and DNA repair, and that the highly expressed TLI genes were enriched in the cell differentiation and methyltransferase activity pathways. This study, the first to use transcriptome analysis to compare SI and TLI, will serve as a basis for assessing the safety of TLI application in assisted reproductive technology (ART).

\section{Introduction}

Since the inception of clinical in vitro fertilization (IVF), assisted reproductive technology (ART) laboratories have focused on improving embryo culture systems. There have been significant developments in ART procedures over the past two decades, including the use of new culture medium (Gardner \& Lane 1997; Mantikou et al. 2013b; Summers \& Biggers 2003), the 
43 change from a 2-3 day culture duration to a 5-6 day culture duration (Marek et al. 1999;

44 Papanikolaou et al. 2006), and the application of hypoxic medium that reportedly increases

embryonic development and clinical outcomes (Bontekoe et al. 2012; Kirkegaard et al. 2013a;

Mantikou et al. 2013a). Additionally, there has been a switch from static to dynamic culture systems, including tilting embryo culture, microfluidic culture, and mechanical vibration during culture (Kim et al. 2009; Matsuura et al. 2010). Although numerous new ART techniques continue to be developed, proper validation of their safety is necessary.

Time-lapse imaging (TLI) is one of the most recently developed ART embryo culture systems. The TLI system permits the continuous evaluation of embryonic development and provides more information of the process (Armstrong et al. 2015; Racowsky et al. 2015). Several studies have claimed that the parameters obtained via TLI can be used for predicting the blastocyst formation, implantation, and pregnancy (Cruz et al. 2012; Kaser \& Racowsky 2014; Kirkegaard et al. 2013b; Meseguer et al. 2011; Meseguer et al. 2012; Wong et al. 2010; Wu et al. 2016). Furthermore, since TLI can assess embryos without removing them, embryos are not exposed to changes in temperature, humidity, light, gas concentrations, and $\mathrm{pH}$, which can impair embryonic development (Fujiwara et al. 2007; Zhang et al. 2010). However, few studies have compared the performances of TLI culture systems to standard incubators (SI) (Alhelou et al. 2018; Barberet et al. 2018; Sciorio et al. 2018; Wu et al. 2017). Our previous study showed that TLI had a higher transferable embryo ratio than the SI group on Day 3 (Wu et al. 2017), which was consistent with other studies (Alhelou et al. 2018; Barberet et al. 2018; Sciorio et al. 2018). This suggests that there are differences between SI- and TLI-cultured embryos, indicating the importance of further 
exploring the molecular mechanisms and pathways underlying these variations.

Pre-implantation embryos are special cells that carry out the most dramatic genome-wide changes of gene expression (Xue et al. 2013; Yan et al. 2013). They are sensitive to their environment, particularly four- and eight-cell embryos in the process of embryonic genome activation (EGA) (Deshmukh et al. 2011; Driver et al. 2012; Gad et al. 2012; Urrego et al. 2017), and require accurate molecular regulation. Environmental changes disturb the normal gene expression that is essential for successful pre-implantation embryonic development (PED) (Sirard 2017; Urrego et al. 2014). For instance, oxygen concentrations outside the incubator can cause the decrease of GLUT1, GLUT3, and VEGF in embryos (Kind et al. 2005), suggesting that these genes are sensitive to environmental changes. Several studies have focused on improving culture conditions and reducing defects that might lead to transcription changes. RNA sequencing (RNASeq) can help determine whether there are differences in the transcriptomic profiles of human embryos cultured in TLI and SI.

In this study, we explored the influence of SI and TLI incubator culturing on the gene expression patterns of Day 3 embryos, and their subsequent influence on pathways and biological functions controlling human embryonic development. Our research provides the first comprehensive datasets for human eight-cell embryos cultured in SI and TLI using RNA-Seq, and the results will serve as a basis for assessing the safety of TLI application in ART. 


\section{Ethics statement}

86

This study was approved by the Institutional Review Board (IRB) of Chongqing Health Center for Women and Children (2016-RGI-01). We followed the guiding principles from the Ministry of Science and Technology (MOST) for the review and approval of human genetic resources. All donor couples voluntarily donated embryos after signing written informed consent at the Chongqing Reproductive and Genetics Institute in the Chongqing Health Center for Women and Children.

\section{Intracytoplasmic sperm injection (ICSI)}

We performed ICSI within 5 hours of oocyte retrieval under an inverted microscope (Olympus IX70, Olympus Optical Co. Ltd., Tokyo, Japan) with a micromanipulation system (CellTram® 4r, Eppendorff, Hamburg, Germany). During ICSI, we placed the oocytes in pre-equilibrated culture droplets and covered them with $6 \mathrm{ml}$ of mineral oil (Ovoil, Vitrolife, Gothenburg, Sweden). We used a holding pipette to position the oocyte. The sperm was injected into the cytoplasm with a micropipette when the first polar body reached the 6 or 12 o'clock position.

\section{Embryo culture}

For the SI group, we placed the injected oocytes into a pre-equilibrated culture dish (Thermo Scientific, Waltham, MA, USA) with $25 \mu \mathrm{L}$ of culture droplets (Vitrolife Sweden AB, Gothenburg, Sweden) covered with $1.2 \mathrm{~mL}$ of paraffin oil (Vitrolife Sweden AB, Sweden). The embryos were cultured in an SI (Sanyo, MCO-5M, Osaka, Japan) at $37^{\circ} \mathrm{C}$ with $5 \% \mathrm{O}_{2}$ and $6 \% \mathrm{CO}_{2}$ 
106

107

108

109

110

111

112

113

114

115

116

117

118

119

120

121

122

123

124

125

126

until embryo transfer on Day 3.

For the TLI group, we transferred the injected oocytes into pre-equilibrated Embryoslides and cultured them in the EmbryoScope ${ }^{\mathrm{TM}}$ until the time of transfer. The embryos cultured in the EmbryoScope $^{\mathrm{TM}}$ had the same culture conditions as the SI group.

\section{Embryo scoring}

We performed morphological assessments of the SI- and TLI-cultured embryos at the same time points using the same criteria. Additional information obtained during TLI was not used for embryo assessment or selection. In this study, the time points for morphological assessments followed the guidelines suggested by the ESHRE/Alpha consensus (31). The embryos were assessed at $17 \pm 1,44 \pm 1$, and $68 \pm 1 \mathrm{~h}$ after ICSI. We scored embryos on their blastomere shape, blastomere number, and fragmentation rate. An embryo was defined as grade 1 when it had an even blastomere shape and $<10 \%$ fragmentation, grade 2 when it had uneven blastomeres and 10 $25 \%$ fragmentation, grade 3 when it had uneven blastomeres and $25-35 \%$ fragmentation, and not recommended for transfer when the fragmentation $>35 \%$. Generally, if one patient have more than eight embryos with grade 1 3, we will transfer two embryos with top quality, and freeze the other top six embryos. Then, the other embryos excluding the eight embryos were defined as not reach the transfer degree in this cycle, even if embryos with grade 3 . However, if one patient did not have enough embryos with grade 1 or 2 , for example, only 1 embryo with grade 3 . We will only transfer one embryo with grade 3 . Here, the embryos used in this study were all from the first situation, and were grade 3 with 8 blastomere. 
128 Collecting human zygotes and eight-cell embryos

129 The patients who donated embryos were between 25 and 30 years old without a history of genetic

130 diseases or smoking. We collected zygotes $6 \mathrm{~h}$ after ICSI, and eight-cell embryos on Day 3. Each

131 couple donated only one zygote or embryo. To avoid possible embryo heterogeneity, each RNA-

132 Seq pool had five zygotes or embryos from five different patients. We processed a total of 15

133 zygotes and 30 embryos (15 TLI-cultured and 15 SI-cultured) for RNA-Seq. All embryos were

134 frozen using vitrification and were then stored. The embryos were cultured for $2 \mathrm{~h}$ after thawing,

135 briefly exposed to an acidic PBS solution for 5-10 s to remove the zona pellucida, and were washed

136 three times in PBS. After all the embryos in one group were prepared, we removed them from the

137 PBS, and immediately placed five embryos per tube into lysis buffer. All embryos used in this

138 study exhibited normal morphology without reaching the transfer degree in this cycle.

\section{RNA sequencing library generation}

141 We performed amplification using the Smart-Seq2 method. We used the Qubit ${ }^{\circledR} 3.0$ Fluorometer and Agilent 2100 Bioanalyzer to check the quality of the cDNA product and to ensure its length was around 1-2 kb. The library was prepared following the manufacturer's instructions (Illumina.

144 Cat. FC-131-1024). After library preparation, we checked the library quality using the PerkinElmer LabChip ${ }^{\circledR}$ GX Touch and Step OnePlus ${ }^{\mathrm{TM}}$ Real-Time PCR System. The libraries were then sequenced on the Illumina HiSeq 4000 with a 150-bp paired-end. 


\section{RNA sequence data processing}

We used Trim_Galore to remove raw sequence reads that contained adapter contamination and poor-quality reads with low PHRED scores. Tophat (version 2.4.0) was used to map all data to the hg38 genome. We used RSEM (version 1.3.0) to calculate the gene expression levels based on the GRCh38.92 annotation database. We performed differential expression analysis using DESeq2, and only kept genes with significantly higher changes $(\log 2$ fold changes $\geq 1$ and false discovery rates $($ FDR $)<0.05)$. We deposited all RNA-Seq data in the NCBI Gene Expression Omnibus (GEO) under accession number GSE114771.

\section{Gene ontology analysis}

We performed gene ontology (GO) analysis using the DAVID web-tool. Biological process, molecular function, and cellular component terms were selected as the background gene sets.

\section{RT-qPCR}

Total RNA extraction, reverse transcription, and pre-amplification were performed using the Single Cell-to-CT qRT-PCR Kit (4458237, Invitrogen, Carlsbad, CA, USA) according to the

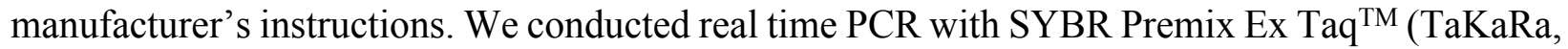
Kyoto, Japan) and the CFX96 Touch Real-Time PCR System (Bio-Rad, Hercules, CA, USA). We measured Ct values using Sequence Detection System software (Bio-Rad, Hercules, CA, USA), and analyzed the gene expression level using the $2^{-\Delta \Delta C t}$ method. All experiments contained at least three biological replicates. We used of the average of GAPDH and HPRT for normalizing RT- 
169

170

171

172

173

175

176

177

178

179

180

181

182

183

qPCR data. The primers used in this study are shown in Table S1.

\section{Statistical analysis}

We used SPSS statistical software 19.0 for the statistical analyses, and one-way ANOVA to assess the differences between the two groups. For all analyses, differences were considered significant when $\mathrm{P}<0.05$.

\section{Results}

\section{Deep sequencing of the human embryo transcriptome}

Using RNA-Seq technology, we studied the transcriptomic profiles of the three groups of human embryos: zygotes, SI-cultured, and TLI-cultured eight-cell embryos. After removing reads with adaptors, reads containing poly $\mathrm{N}$, and low-quality reads from the raw data, a total of $68,950,172(88.1 \%$ of the raw data), $32,041,678(72.35 \%)$, and $27,625,662(62.63 \%)$ trimmed reads remained in the zygote group; 65,996,908 (88.94\%), 41,141,300 (70.82\%), and 52,110,290 $(83.02 \%)$ remained in the SI group; and 53,242,892 $(80.21 \%), 45,191,346(81.37 \%)$, and $39,410,428(81.86 \%)$ remained in the TLI group, respectively (Table 1).

We analyzed the trimmed reads via TopHat for alignment with the reference genome (hg38). We mapped approximately 400 million paired-end reads derived from the nine samples, with a mapping rate ranging from $89 \%$ to $93 \%$ (Table 1). The typical number of detectable genes in the individual samples ranged from approximately 18,000 to 27,000 genes. 
190

191

192

193

194

195

196

197

198

199

200

201

202

203

204

205

206

207

208

209

210

\section{Global transcriptomic profile analysis}

The number of trimmed reads mapped to a gene was normalized to transcripts per kilobase million (TPM) values to accurately measure the gene expression level. To determine whether the TLI- and SI-cultured embryos had distinct transcriptomic landscapes, we analyzed the RNA-Seq data using unsupervised hierarchical clustering. We included all genes that were expressed in at least one of the nine samples with TPM values $\geq 0.1$. The results showed that embryos in the same developmental stage clustered together. Moreover, the TLI samples were similar to the SI samples and were distinct from the zygotes (Figure 1A). Our principal component analysis (PCA) also found these distinct expression patterns (Figure 1B).

\section{Differentially expressed gene analysis}

Although the global transcriptomic profiles of the TLI group were more similar to those of the SI group than the zygotes, we could not distinguish any small molecular differences between TLIand SI-cultured embryos. Therefore, we independently compared the TLI and SI groups and identified a total of 539 differentially expressed genes (DEGs, FDR $<0.05,>2$-fold change). We verified the accuracy using cluster analysis, which successfully distinguished the TLI from the SI group (Figure 2A). Of the 539 DEGs, 24 genes were exclusively expressed in the TLI group, and 39 genes were exclusively expressed in the SI group. Of the remaining 476 genes, 188 showed higher expression in the TLI group while 288 genes showed higher expression in the SI group (Table S2). We also detected the differential expression of solute carrier family (SLC) genes, including $S L C 5 A 12, S L C 9 B 2$, and $S L C 35 A 3$, which play important roles in early embryonic 
211 development (Song et al. 2005). Figure 2B shows the fold-change breakdown across the DEGs of 212 the two groups.

\section{Functional analysis}

To identify the functions of DEGs, we performed GO enrichment analysis of SI- and TLIoverexpressed genes. In SI-overexpressed genes, we found many more enriched GO terms, particularly biological process terms (83\%, Figure 3). The overexpressed genes in SI tended to execute functions, such as transcription, RNA splicing, and DNA repair. However, the overexpressed genes in TLI belonged to several different categories, including the cell differentiation and methyltransferase activity pathways. In spite of the similarities between the SI and TLI groups, there were still small differences that could influence specific pathways and molecular functions during embryonic development.

\section{Validating differentially expressed genes}

To verify the reliability of our sequencing data, we selected four upregulated and downregulated genes from several enriched pathways, including RNA splicing, DNA repair, cell differentiation, and methyltransferase activity. We used qPCR to analyze their expression levels in eight-cell embryos cultured in SI and TLI. We detected the upregulated expressions of RBM14,

(Figure 4). These results were consistent with our sequencing data. 
232

233

234

235

236

237

238

239

240

241

242

243

244

245

246

247

248

249

250

251

252

\section{Discussion}

There has been a longstanding debate regarding the superiority of the closed culture system over the SI system. To settle this debate, the safety of the closed TLI system first needs to be confirmed.

Previous studies that have compared TLI and SI focused on embryonic development and clinical outcomes (Barberet et al. 2018; Chen et al. 2017; Kaser \& Racowsky 2014; Meseguer et al. 2012; Park et al. 2015; Wu et al. 2017; Wu et al. 2016). However, none have explored whether TLI and SI culturing results in transcriptomic alterations in embryos. We performed the first transcriptomic analysis comparing the gene expression profiles of SI- and TLI-cultured human embryos. Our results showed similarities between the global transcriptomic profiles of the SI and TLI groups.

TLI has two main advantages: (i) it can provide a more stable in vitro culture environment for embryo development, and (ii) using continuous imaging, TLI offers embryologists more embryo morphokinetic parameters which can improve the efficiency of embryo selection in ART. In this study, we only focused on the first advantage. To accurately compare the two culture systems, we selected embryos of equivalent morphological quality in the TLI and SI groups. The embryos were cultured in individual medium droplet. Additionally, we did not use the morphokinetic parameters provided by TLI for embryo assessment, and we assessed the embryos in both groups at the same time points using the same criteria.

Embryos are sensitive to microenvironmental changes during in vitro culture. Embryonic developmental kinetics, transcription, proteomes, metabolic state, histone remodeling, and methylation patterns have been shown to change when exposed to atmospheric oxygen (Harvey et al. 2004; Katz-Jaffe et al. 2005; Li et al. 2016; Takahashi et al. 2000; Van Soom et al. 2002). 
253 Opening the incubator door disrupts the $\mathrm{CO}_{2}$ tension of the embryo culture microenvironment, 254 temporarily changing the $\mathrm{pH}$ of the culture medium and negatively affecting the embryo. A 255 previous study reported that during SI culturing, temperature recovery took approximately $30 \mathrm{~min}$ 256 after a $5 \mathrm{~s}$ door opening/closing procedure, and the recovery of oxygen tension took about $7.8 \pm$ 0.9 min (Fujiwara et al. 2007). Exposure to visible light can also create additional stress for embryos (Ottosen et al. 2007). Nonetheless, the morphological assessment of embryos is a vital step in a typical ART program. This method requires moving embryos outside the incubator for microscopic observation once daily from Day 1 to Day 3 after fertilization. Most laboratories generally culture 10 dishes in one SI, so there are at least 30 door opening/closing procedures when

SI culturing. The dish is removed only once in TLI. Zhang et al. (2010) reported that decreasing the number of embryo observation times could significantly improve the blastocyst development rate. Although TLI allows for the continuous imaging of embryonic development in an undisturbed culture system, the long exposure to light during TLI might affect normal gene expression in embryos.

Although the two embryo groups were of similar morphological grades, they may have had different transcriptomes. Since our primary objective was to investigate the differences between the transcriptomic landscapes of TLI- and SI-cultured embryos, we pooled five embryos in one sample to balance the heterogeneity from different patients. In total, we identified 539 genes showing differential expression, and 284 genes with greater than 10-fold differences in gene expression level. We distinguished these two groups using hierarchical clustering, which showed that the embryos from the two groups had small molecular differences. We hypothesized that the 
274

275

276

277

278

279

280

281

282

283

284

285

286

transcriptional changes in the embryos were caused by a consistent directional response to the different culture microenvironments.

In addition to the individual gene differences, our study also revealed several significantly enriched GO terms. One of particular interest was the RNA splicing pathway, which contains 14 genes with high expression in the SI group. Alternative splicing is one type of gene regulatory mechanism at the RNA level that ensures proper transcriptome regulation in PED, especially during EGA. Our previous study showed that the overexpression of HNRPU, which acts as an alternative splicing regulator, can affect the embryonic development of mice (Wang et al. 2016). We found that SI-cultured embryos displayed a higher expression of HNRPUL1, a HNRPU-like gene. The gene ELAVL1, which can bind to AU-rich genes including SLC genes and whose activity is regulated by the p38 MAPK pathway, also showed high expression in the SI group (Calder et al. 2011; Fan \& Steitz 1998; Peng et al. 1998; Song et al. 2005). Previous studies have found that the inhibition of the p38 MAPK pathway can block PED and decrease the expression of SLC genes (Song et al. 2005). In accordance with these results, we found downregulation of several SLC genes, including $S L C 5 A 12$, SLC9B2, and SLC35A3, in the SI group. We postulated that the different environments in SI may have disrupted normal alternative splicing regulation during human EGA. In the SI group, we found higher expressions of other genes involved in DNA repair, including DOT1L, MMS19, POLL, SMARCB1, POLE, CEP164, TRRAP, and RBM14. The activation of these genes might have been linked to oxidative stress or an aberrant DNA damage response, both of which affect long-term embryo viability (Ooga et al. 2013). There is evidence that a low expression of DOTIL is required for PED and heterochromatin remodeling (Phillips et 
295 al. 2016; Tao et al. 2017). Additionally, we detected a few genes in the TLI group with high

296

297

298

299

300

301

302

303

304

305

306

307

308

309

310

311

312

313

314

315

expressions of ACTN1, MAP1A, CFL1, FLNB, DNASE1, WHAMM, PFN1, and SYNE2 related to the light stimulus response despite the lack of significant enrichment. Although it has been reported that exposure to light during TLI observations does not affect PED (Nakahara et al. 2010), the potential long-term effects have not been studied. Our results showed that in spite of their similarities, the differences between the two groups could affect particular pathways and important molecular functions of the embryos.

The limitations of this study are worth mentioning. The embryos used in this study were not top grade, and it is known that early embryos can self-correct during subsequent development (Coticchio et al. 2019). Therefore, future studies should further explore the differences between blastocysts cultured in SI and TLI.

\section{Conclusion}

This is the first study to compare the transcriptome of embryos cultured in closed TLI and SI. Although the global transcriptomic profiles were similar between the SI and TLI groups, their distinct culture microenvironments produced several different results. The impact of these small differences in gene expression on embryonic development requires further research. Our study provides a basis to understanding the molecular mechanisms underlying these differences, and represents a new form of research on the safety of TLI in ART. 
316

317

318

319

320

321

322

323

324

325

326

327

328

329

330

331

332

333

334

335

336

\section{References}

Alhelou Y, Mat Adenan NA, and Ali J. 2018. Embryo culture conditions are significantly improved during uninterrupted incubation: A randomized controlled trial. Reprod Biol 18:40-45. 10.1016/j.repbio.2017.12.003

Armstrong S, Vail A, Mastenbroek S, Jordan V, and Farquhar C. 2015. Time-lapse in the IVF-lab: how should we assess potential benefit? Hum Reprod 30:3-8. 10.1093/humrep/deu250

Barberet J, Chammas J, Bruno C, Valot E, Vuillemin C, Jonval L, Choux C, Sagot P, Soudry A, and Fauque P. 2018. Randomized controlled trial comparing embryo culture in two incubator systems: G185 K-System versus EmbryoScope. Fertil Steril 109:302-309 e301. 10.1016/j.fertnstert.2017.10.008

Bontekoe S, Mantikou E, van Wely M, Seshadri S, Repping S, and Mastenbroek S. 2012. Low oxygen concentrations for embryo culture in assisted reproductive technologies. Cochrane Database Syst Rev:CD008950. 10.1002/14651858.CD008950.pub2

Calder MD, Watson PH, and Watson AJ. 2011. Culture medium, gas atmosphere and MAPK inhibition affect regulation of RNA-binding protein targets during mouse preimplantation development. Reproduction 142:689-698. 10.1530/REP-11-0082

Chen M, Wei S, Hu J, Yuan J, and Liu F. 2017. Does time-lapse imaging have favorable results for embryo incubation and selection compared with conventional methods in clinical in vitro fertilization? A meta-analysis and systematic review of randomized controlled trials. PLoS One 12:e0178720. 10.1371/journal.pone.0178720 
337 Coticchio G, Lagalla C, Sturmey R, Pennetta F, and Borini A. 2019. The enigmatic morula:

338 mechanisms of development, cell fate determination, self-correction and implications for ART.

339 Hum Reprod Update 25:422-438. 10.1093/humupd/dmz008

340 Cruz M, Garrido N, Herrero J, Perez-Cano I, Munoz M, and Meseguer M. 2012. Timing of cell

341 division in human cleavage-stage embryos is linked with blastocyst formation and quality. Reprod

342 Biomed Online 25:371-381. 10.1016/j.rbmo.2012.06.017

343 Deshmukh RS, Ostrup O, Ostrup E, Vejlsted M, Niemann H, Lucas-Hahn A, Petersen B, Li J,

344 Callesen H, and Hyttel P. 2011. DNA methylation in porcine preimplantation embryos developed

345 in vivo and produced by in vitro fertilization, parthenogenetic activation and somatic cell nuclear

346 transfer. Epigenetics 6:177-187. 13519 [pii]

347 Driver AM, Penagaricano F, Huang W, Ahmad KR, Hackbart KS, Wiltbank MC, and Khatib H.

348 2012. RNA-Seq analysis uncovers transcriptomic variations between morphologically similar in

349 vivo- and in vitro-derived bovine blastocysts. BMC Genomics 13:118. 10.1186/1471-2164-13-118

350 1471-2164-13-118 [pii]

351 Fan XC, and Steitz JA. 1998. Overexpression of HuR, a nuclear-cytoplasmic shuttling protein,

352 increases the in vivo stability of ARE-containing mRNAs. EMBO $J$ 17:3448-3460.

$353 \quad 10.1093 / \mathrm{emboj} / 17.12 .3448$

354 Fujiwara M, Takahashi K, Izuno M, Duan YR, Kazono M, Kimura F, and Noda Y. 2007. Effect

355 of micro-environment maintenance on embryo culture after in-vitro fertilization: comparison of top-load mini incubator and conventional front-load incubator. J Assist Reprod Genet 24:5-9. 
358 Gad A, Hoelker M, Besenfelder U, Havlicek V, Cinar U, Rings F, Held E, Dufort I, Sirard MA,

359 Schellander K, and Tesfaye D. 2012. Molecular mechanisms and pathways involved in bovine

360 embryonic genome activation and their regulation by alternative in vivo and in vitro culture

361 conditions. Biol Reprod 87:100. 10.1095/biolreprod.112.099697

362 biolreprod.112.099697 [pii]

363 Gardner DK, and Lane M. 1997. Culture and selection of viable blastocysts: a feasible proposition

364 for human IVF? Hum Reprod Update 3:367-382.

365 Harvey AJ, Kind KL, Pantaleon M, Armstrong DT, and Thompson JG. 2004. Oxygen-regulated

366 gene expression in bovine blastocysts. Biol Reprod 71:1108-1119. 10.1095/biolreprod.104.028639

367 Kaser DJ, and Racowsky C. 2014. Clinical outcomes following selection of human

368 preimplantation embryos with time-lapse monitoring: a systematic review. Hum Reprod Update

369 20:617-631. 10.1093/humupd/dmu023

370 dmu023 [pii]

371 Katz-Jaffe MG, Linck DW, Schoolcraft WB, and Gardner DK. 2005. A proteomic analysis of

372 mammalian preimplantation embryonic development. Reproduction 130:899-905.

373 10.1530/rep.1.00854

374 Kim MS, Bae CY, Wee G, Han YM, and Park JK. 2009. A microfluidic in vitro cultivation system

375 for mechanical stimulation of bovine embryos. Electrophoresis 30:3276-3282.

$376 \quad 10.1002 /$ elps.200900157

377 Kind KL, Collett RA, Harvey AJ, and Thompson JG. 2005. Oxygen-regulated expression of

378 GLUT-1, GLUT-3, and VEGF in the mouse blastocyst. Mol Reprod Dev 70:37-44. 
379

380

381

382

383

384

385

386

387

388

389

390

391

392

393

394

395

396

397

398

399

$10.1002 / \mathrm{mrd} .20183$

Kirkegaard K, Hindkjaer JJ, and Ingerslev HJ. 2013a. Effect of oxygen concentration on human embryo development evaluated by time-lapse monitoring. Fertil Steril 99:738-744 e734.

\subsection{6/j.fertnstert.2012.11.028}

Kirkegaard K, Kesmodel US, Hindkjaer JJ, and Ingerslev HJ. 2013b. Time-lapse parameters as predictors of blastocyst development and pregnancy outcome in embryos from good prognosis patients: a prospective cohort study. Hum Reprod 28:2643-2651. 10.1093/humrep/det300

Li W, Goossens K, Van Poucke M, Forier K, Braeckmans K, Van Soom A, and Peelman LJ. 2016. High oxygen tension increases global methylation in bovine 4-cell embryos and blastocysts but does not affect general retrotransposon expression. Reprod Fertil Dev 28:948-959. 10.1071/RD14133

Mantikou E, Bontekoe S, van Wely M, Seshadri S, Repping S, and Mastenbroek S. 2013a. Low oxygen concentrations for embryo culture in assisted reproductive technologies. Hum Reprod Update 19:209. 10.1093/humupd/dms055

Mantikou E, Youssef MA, van Wely M, van der Veen F, Al-Inany HG, Repping S, and Mastenbroek S. 2013b. Embryo culture media and IVF/ICSI success rates: a systematic review.

Hum Reprod Update 19:210-220. 10.1093/humupd/dms061

Marek D, Langley M, Gardner DK, Confer N, Doody KM, and Doody KJ. 1999. Introduction of blastocyst culture and transfer for all patients in an in vitro fertilization program. Fertil Steril 72:1035-1040.

Matsuura K, Hayashi N, Kuroda Y, Takiue C, Hirata R, Takenami M, Aoi Y, Yoshioka N, Habara 
400

401

402

403

404

405

406

407

408

409

410

411

412

413

414

415

416

417

418

419

420

T, Mukaida T, and Naruse K. 2010. Improved development of mouse and human embryos using a tilting embryo culture system. Reprod Biomed Online 20:358-364. 10.1016/j.rbmo.2009.12.002

Meseguer M, Herrero J, Tejera A, Hilligsoe KM, Ramsing NB, and Remohi J. 2011. The use of morphokinetics as a predictor of embryo implantation. Hum Reprod 26:2658-2671.

\subsection{3/humrep/der256}

Meseguer M, Rubio I, Cruz M, Basile N, Marcos J, and Requena A. 2012. Embryo incubation and selection in a time-lapse monitoring system improves pregnancy outcome compared with a standard incubator: a retrospective cohort study. Fertil Steril 98:1481-1489 e1410. 10.1016/j.fertnstert.2012.08.016

Nakahara T, Iwase A, Goto M, Harata T, Suzuki M, Ienaga M, Kobayashi H, Takikawa S, Manabe S, Kikkawa F, and Ando H. 2010. Evaluation of the safety of time-lapse observations for human embryos. J Assist Reprod Genet 27:93-96. 10.1007/s10815-010-9385-8

Ooga M, Suzuki MG, and Aoki F. 2013. Involvement of DOT1L in the remodeling of heterochromatin configuration during early preimplantation development in mice. Biol Reprod 89:145. 10.1095/biolreprod.113.113258

Ottosen LD, Hindkjaer J, and Ingerslev J. 2007. Light exposure of the ovum and preimplantation embryo during ART procedures. J Assist Reprod Genet 24:99-103. 10.1007/s 10815-006-9081-x

Papanikolaou EG, Camus M, Kolibianakis EM, Van Landuyt L, Van Steirteghem A, and Devroey P. 2006. In vitro fertilization with single blastocyst-stage versus single cleavage-stage embryos. $N$ Engl J Med 354:1139-1146. 10.1056/NEJMoa053524

Park H, Bergh C, Selleskog U, Thurin-Kjellberg A, and Lundin K. 2015. No benefit of culturing 
421 embryos in a closed system compared with a conventional incubator in terms of number of good 422 quality embryos: results from an RCT. Hum Reprod 30:268-275. 10.1093/humrep/deu316

423 Peng SS, Chen CY, Xu N, and Shyu AB. 1998. RNA stabilization by the AU-rich element binding 424 protein, HuR, an ELAV protein. EMBO J 17:3461-3470. 10.1093/emboj/17.12.3461

425 Phillips TC, Wildt DE, and Comizzoli P. 2016. Incidence of methylated histones H3K4 and $426 \mathrm{H} 3 \mathrm{~K} 79$ in cat germinal vesicles is regulated by specific nuclear factors at the acquisition of 427 developmental competence during the folliculogenesis. J Assist Reprod Genet 33:783-794. 10.1007/s10815-016-0706-4

Racowsky C, Kovacs P, and Martins WP. 2015. A critical appraisal of time-lapse imaging for embryo selection: where are we and where do we need to go? J Assist Reprod Genet 32:10251030. $10.1007 / \mathrm{s} 10815-015-0510-6$

Sciorio R, Thong JK, and Pickering SJ. 2018. Comparison of the development of human embryos cultured in either an EmbryoScope or benchtop incubator. J Assist Reprod Genet 35:515-522.

Sirard MA. 2017. The influence of in vitro fertilization and embryo culture on the embryo epigenetic constituents and the possible consequences in the bovine model. J Dev Orig Health Dis $8: 411-417.10 .1017 / \mathrm{S} 2040174417000125$

Song IS, Tatebe S, Dai W, and Kuo MT. 2005. Delayed mechanism for induction of gammaglutamylcysteine synthetase heavy subunit mRNA stability by oxidative stress involving p38 440 mitogen-activated protein kinase signaling. $J$ Biol Chem 280:28230-28240. 
442 Summers MC, and Biggers JD. 2003. Chemically defined media and the culture of mammalian 443 preimplantation embryos: historical perspective and current issues. Hum Reprod Update 9:557444582.

445 Takahashi M, Keicho K, Takahashi H, Ogawa H, Schultz RM, and Okano A. 2000. Effect of 446 oxidative stress on development and DNA damage in in-vitro cultured bovine embryos by comet 447 assay. Theriogenology 54:137-145.

448 Tao J, Zhang Y, Zuo X, Hong R, Li H, Liu X, Huang W, Cao Z, and Zhang Y. 2017. DOT1L 449 inhibitor improves early development of porcine somatic cell nuclear transfer embryos. PLoS One 12:e0179436. 10.1371/journal.pone.0179436

Urrego R, Bernal-Ulloa SM, Chavarria NA, Herrera-Puerta E, Lucas-Hahn A, Herrmann D,

Winkler S, Pache D, Niemann H, and Rodriguez-Osorio N. 2017. Satellite DNA methylation status and expression of selected genes in Bos indicus blastocysts produced in vivo and in vitro. Zygote 25:131-140. 10.1017/S096719941600040X

Urrego R, Rodriguez-Osorio N, and Niemann H. 2014. Epigenetic disorders and altered gene expression after use of Assisted Reproductive Technologies in domestic cattle. Epigenetics 9:803-

815. 10.4161/epi.28711

Van Soom A, Yuan YQ, Peelman LJ, de Matos DG, Dewulf J, Laevens H, and de Kruif A. 2002.

Prevalence of apoptosis and inner cell allocation in bovine embryos cultured under different oxygen tensions with or without cysteine addition. Theriogenology 57:1453-1465.

Li W, Zhou Q, and Liu Z. 2016. A novel long intergenic noncoding RNA indispensable for the 
463 cleavage of mouse two-cell embryos. EMBO Rep 17:1452-1470. embr.201642051 [pii]

$464 \quad 10.15252 /$ embr.201642051

465 Wong CC, Loewke KE, Bossert NL, Behr B, De Jonge CJ, Baer TM, and Reijo Pera RA. 2010.

466 Non-invasive imaging of human embryos before embryonic genome activation predicts

467 development to the blastocyst stage. Nat Biotechnol 28:1115-1121. 10.1038/nbt.1686

468 nbt.1686 [pii]

469 Wu L, Han W, Wang J, Zhang X, Liu W, Xiong S, Han S, Liu J, Gao Y, and Huang G. 2017.

470 Embryo culture using a time-lapse monitoring system improves live birth rates compared with a

471 conventional culture system: a prospective cohort study. Hum Fertil (Camb):1-8.

$47210.1080 / 14647273.2017 .1335886$

473 Wu L, Han W, Zhang X, Wang J, Liu W, Xiong S, and Huang G. 2016. A retrospective analysis

474 of morphokinetic parameters according to the implantation outcome of IVF treatment. Eur J Obstet

475 Gynecol Reprod Biol 197:186-190. 10.1016/j.ejogrb.2015.12.002

476 S0301-2115(15)00446-7 [pii]

477 Xue Z, Huang K, Cai C, Cai L, Jiang CY, Feng Y, Liu Z, Zeng Q, Cheng L, Sun YE, Liu JY,

478 Horvath S, and Fan G. 2013. Genetic programs in human and mouse early embryos revealed by

479 single-cell RNA sequencing. Nature 500:593-597. 10.1038/nature12364

480 nature 12364 [pii]

481 Yan L, Yang M, Guo H, Yang L, Wu J, Li R, Liu P, Lian Y, Zheng X, Yan J, Huang J, Li M, Wu

482 X, Wen L, Lao K, Qiao J, and Tang F. 2013. Single-cell RNA-Seq profiling of human

483 preimplantation embryos and embryonic stem cells. Nat Struct Mol Biol 20:1131-1139. 
$484 \quad 10.1038 /$ nsmb. 2660

485 nsmb.2660 [pii]

486 Zhang JQ, Li XL, Peng Y, Guo X, Heng BC, and Tong GQ. 2010. Reduction in exposure of human

487 embryos outside the incubator enhances embryo quality and blastulation rate. Reprod Biomed

488 Online 20:510-515. 10.1016/j.rbmo.2009.12.027

489 


\section{Table $\mathbf{1}$ (on next page)}

Summary of the sequencing qualities and reads mapping. 
1 Table 1 Summary of the sequencing qualities and reads mapping

\begin{tabular}{cccccc}
\hline Sample_ID & Raw reads & $\begin{array}{c}\text { Clean Reads } \\
\text { (after QC) }\end{array}$ & Q30 (\%) & $\begin{array}{c}\text { Mapped } \\
\text { reads }\end{array}$ & Rate (\%) \\
\hline Zygote \#1 & 83071408 & 68950172 & 88.1 & 61702497 & 89.49 \\
Zygote \#2 & 53566316 & 32041678 & 72.35 & 29486629 & 92.03 \\
Zygote \#3 & 63087152 & 27625662 & 62.63 & 25162686 & 91.08 \\
8-cell SI \#1 & 78213756 & 65996908 & 88.94 & 60610716 & 91.84 \\
8-cell SI \#2 & 67695944 & 41141300 & 70.82 & 37656820 & 91.53 \\
8-cell SI \#3 & 62160362 & 52110290 & 83.02 & 47953929 & 92.03 \\
8-cell TLI \#1 & 67586738 & 53242892 & 80.21 & 49691133 & 93.33 \\
8-cell TLI \#2 & 56754526 & 45191346 & 81.37 & 42197537 & 93.38 \\
8-cell TLI \#3 & 48816418 & 39410428 & 81.86 & 36581706 & 92.82 \\
\hline
\end{tabular}




\section{Figure 1}

Global analysis of expression patterns in human zygotes, and 8-cell embryos cultured in SI and TLI .

(A) Unsupervised hierarchical clustering. All genes expressed in at least one of the samples with TPM $\geq 0.1$ were used for the analysis. (B) PCA of the transcriptome of human zygotes (pink), and 8-cell embryos cultured in TLI (blue) and SI (orange). PCA1, PCA2, and PCA3 represent the top three dimensions of the genes showing differential expression among these samples, which accounts for $32.7 \%, 14.5 \%$, and $10.7 \%$ of the expressed genes, respectively.

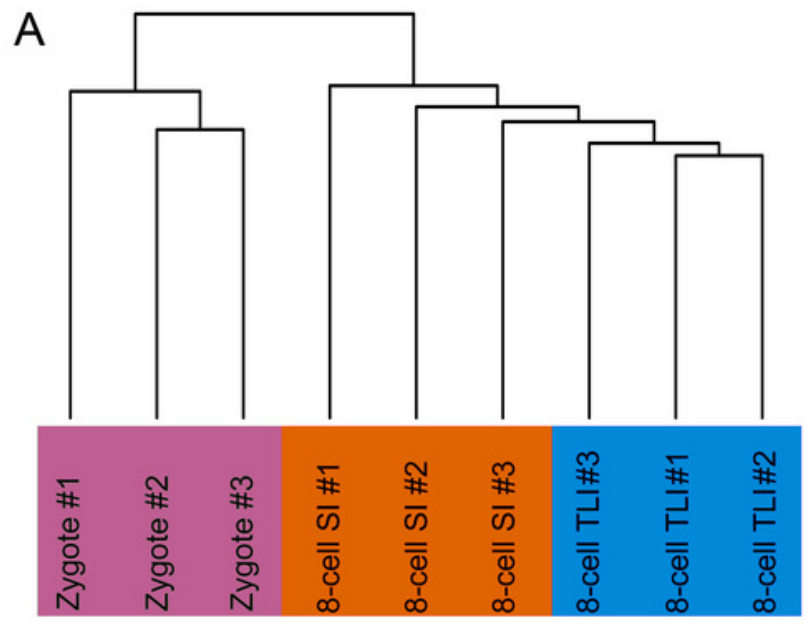

B

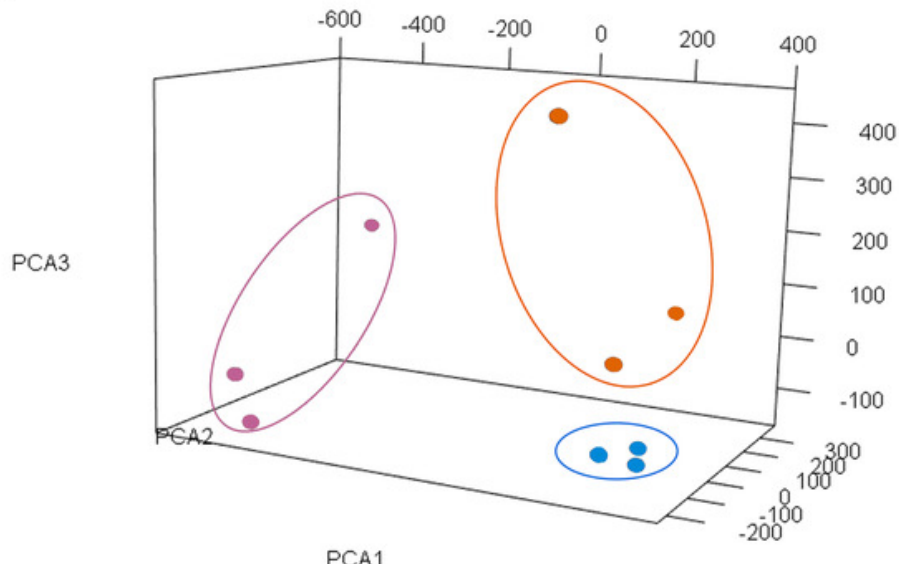


Figure 2

The differentially expressed genes between SI- and in TLI-cultured embryos.

(A) Heatmap and hierarchical cluster of DEGs. (B) Fold-change differences in expression of DEGs.

A

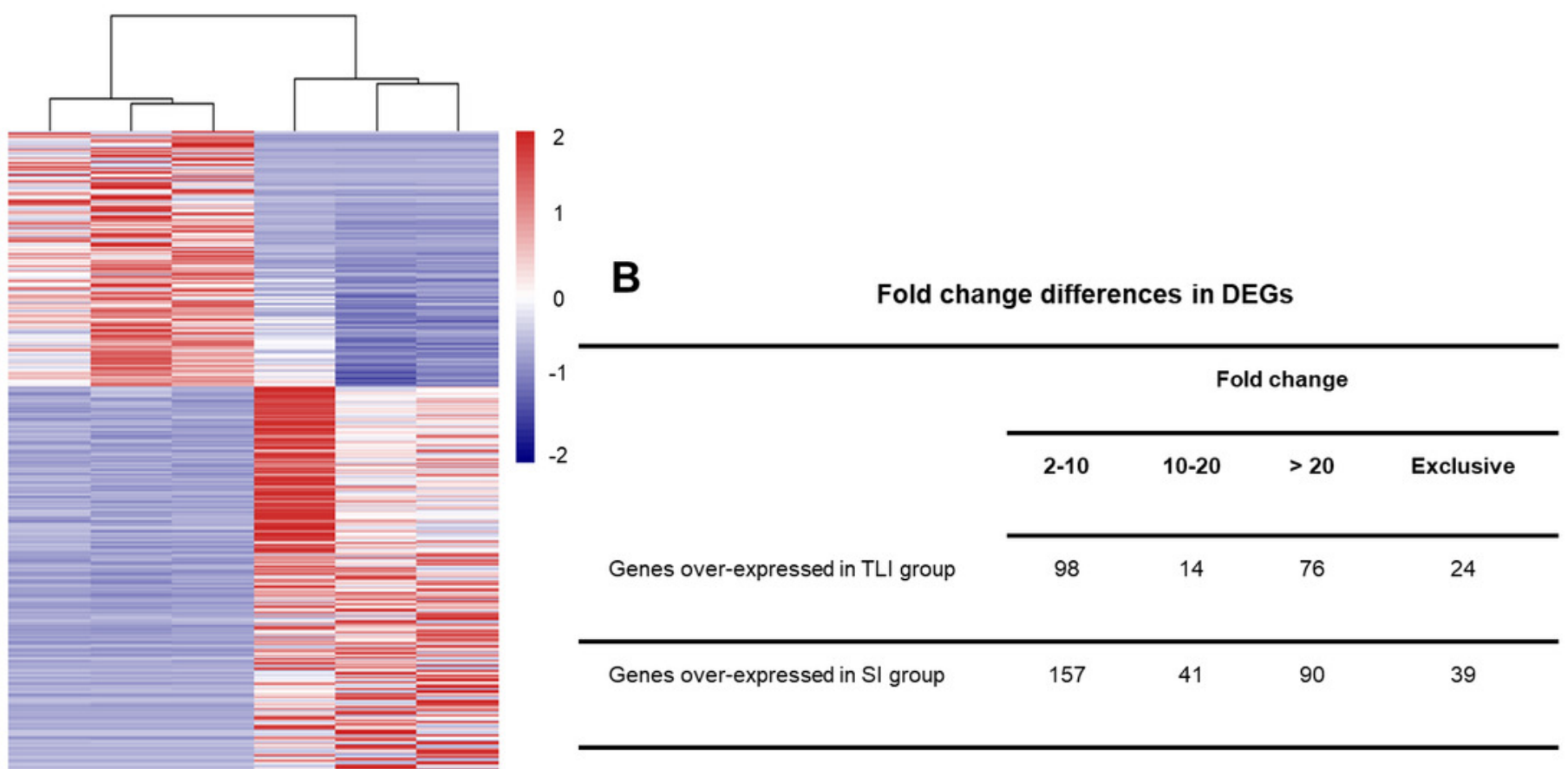

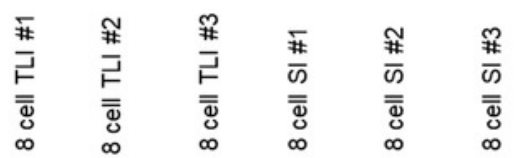


Figure 3

Function enrichment analysis result of DEGs.

The colors indicate the significance ( $P$-value), the size represents the number of genes enriching the corresponding annotation, and the shape shows the sample group. The fold enrichment of analysis is shown in the horizontal axis. (A) "biological process" term. (B) "molecular function" term. (C) "cellular component" term.

A

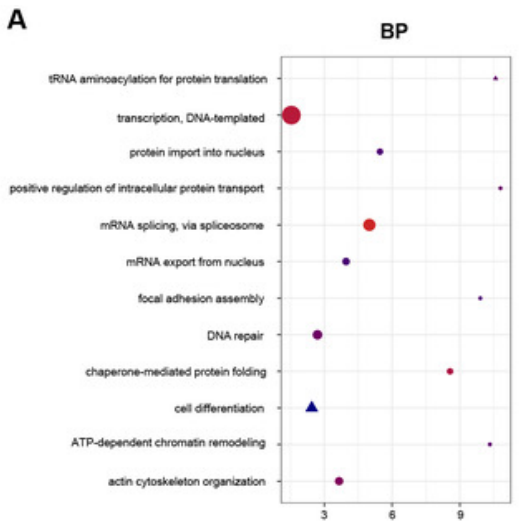

B

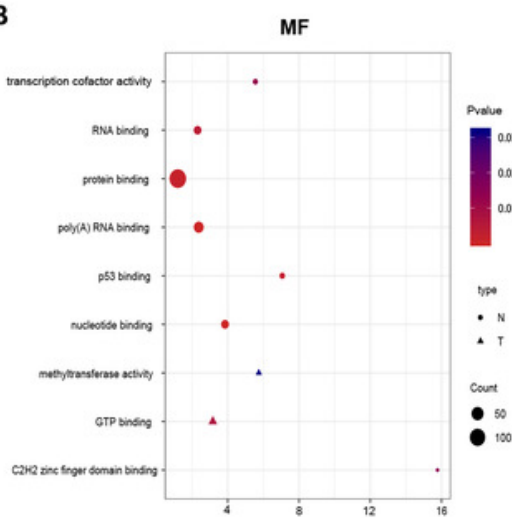

C

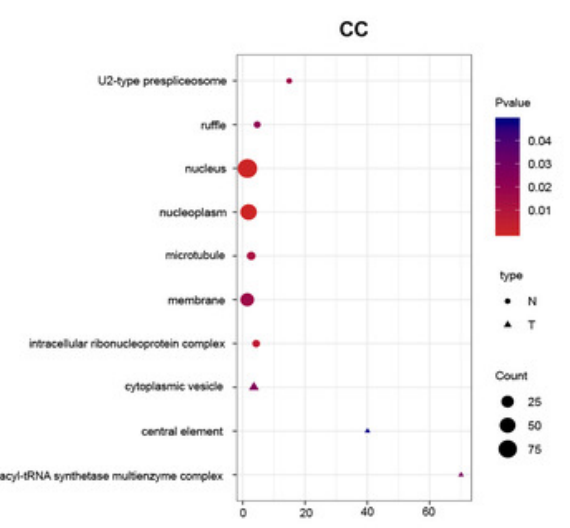


Figure 4

qPCR validation of the eight differentially expressed genes.

(A-D) The upregulated expression level of embryos in SI were confirmed by qPCR. (E-H) The upregulated expression level of embryos in TLI were confirmed by qPCR. The average of the GAPDH and HPRT was used for normalization. Results are presented as mean values \pm SEM. * indicate significant differences $(p<0.05)$.

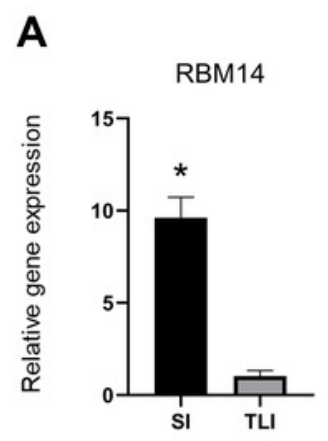

B

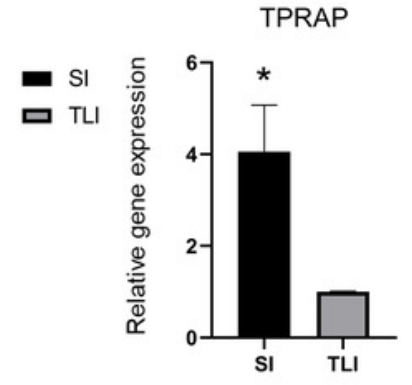

E ANGPT1

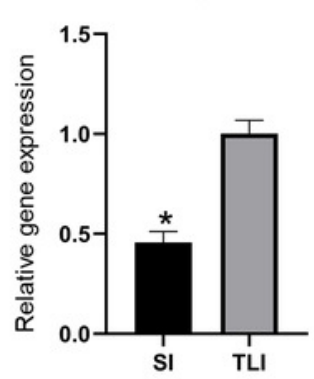

C
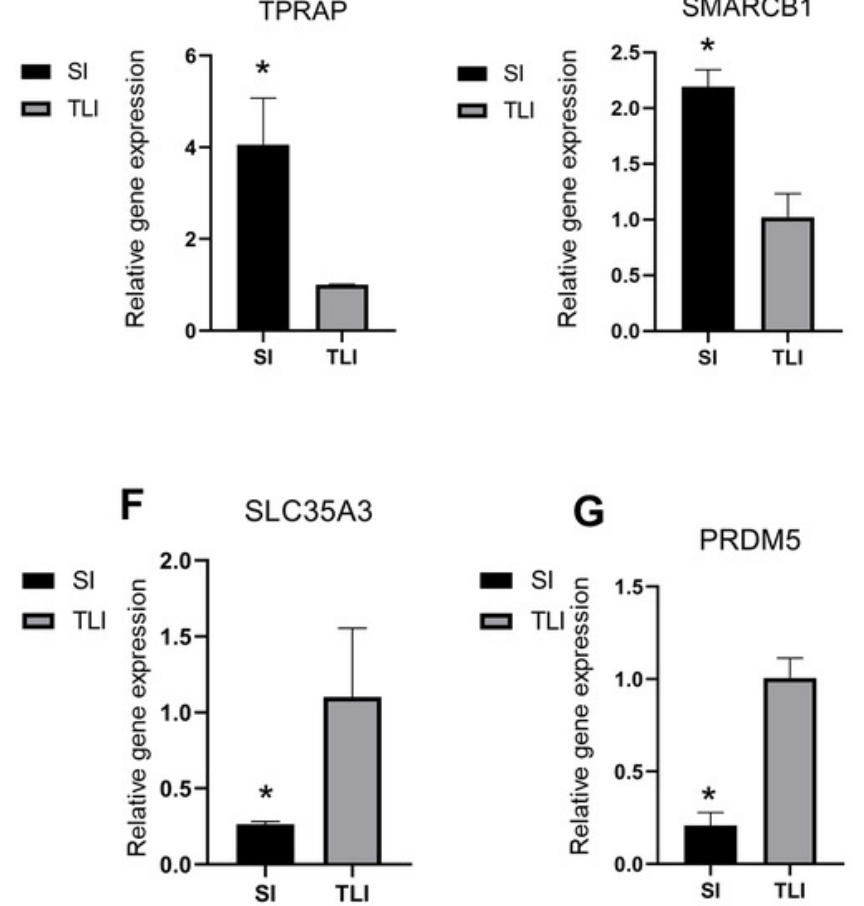

G

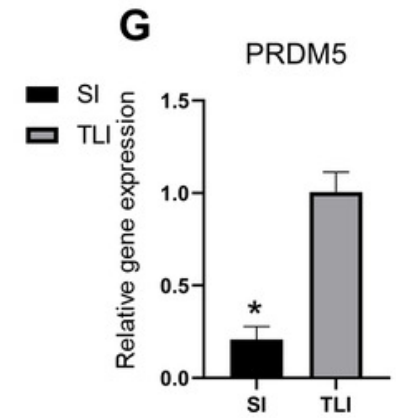

H ZIC3

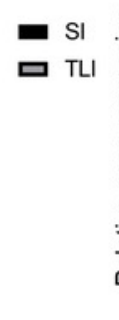

ELAVL1
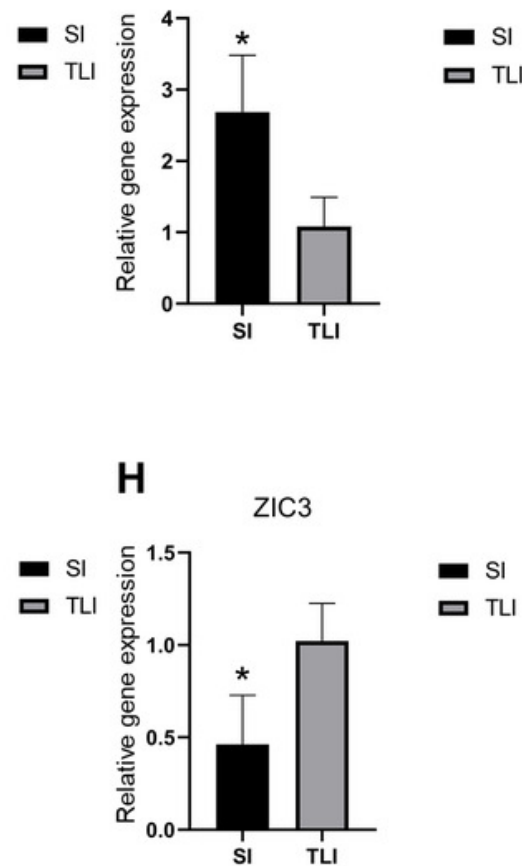